\title{
Increasing the Therapeutic Efficacy of Extracellular Vesicles From the Antigen-Specific Antibody and Light Chain Perspective
}

\author{
Katarzyna Nazimek and Krzysztof Bryniarski * \\ Department of Immunology, Jagiellonian University Medical College, Krakow, Poland
}

Due to their exceptional properties, extracellular vesicles (EVs) receive special attention as next generation biotherapeutics and vehicles for drug delivery. However, despite having many advantages over cell-based therapies, EVs usually exert lower therapeutic efficacy. This results from a number of hurdles that are faced by the EV-based approaches. Administered EVs could be rapidly cleared by the mononuclear phagocytes as well as can randomly distribute within various tissues, making tissue penetration and cell targeting insufficient. However, recent research findings imply that these limitations could be overcome with the use of antigen-specific antibodies and light chains. Major

Edited by:

Eduardo Marbán,

Independent researcher, Los Angeles,

CA, United States

Reviewed by:

Xiaoguang (Margaret) Liu, University of Alabama at Birmingham, United States

Stefano Fais,

National Institute of Health (ISS), Italy

${ }^{*}$ Correspondence:

Krzysztof Bryniarski

mmbrynia@cyf-kr.edu.pl

Specialty section:

This article was submitted to Molecular and Cellular Pathology,

a section of the journal

Frontiers in Cell and Developmental

Biology

Received: 07 October 2021

Accepted: 05 November 2021

Published: 24 November 2021

Citation:

Nazimek K and Bryniarski K (2021) Increasing the Therapeutic Efficacy of

Extracellular Vesicles From the Antigen-Specific Antibody and Light

Chain Perspective.

Front. Cell Dev. Biol. 9:790722.

doi: 10.3389/fcell.2021.790722 histocompatibility complex (MHC) class II-expressing EVs have been shown to form aggregates after co-incubation with antigen-specific antibodies, which greatly enhanced their biological efficacy. On the other hand, EVs could be coated with antibody light chains of chosen specificity to direct them towards desired target cell population. Both findings open up a promising perspective to achieve the highest efficacy of the EV-based approaches. Herein we discuss the opportunities for enhancing extracellular vesicle's biological activity by using specific antibodies and light chains in the context of the challenges faced by such therapeutic approach.

Keywords: antibody, biotherapeutics, exosomes, extracellular vesicles, treatment efficiency

\section{INTRODUCTION}

Extracellular vesicles (EVs) encompass all classes of lipid-membrane vesicles that differ in the formation pathway but are then released by virtually all cells to their surrounding microenvironment (Yáñez-Mó et al., 2015; Pironti et al., 2021). As a newly discovered mood of intercellular communication, they currently receive special attention as next generation biotherapeutics with likely very limited adverse effects of administration (Kalluri and LeBleu, 2020), and multiple advantages over synthetic liposomes (Vader et al., 2016; Nazimek and Bryniarski, 2020b). Accordingly, EVs, exosomes especially, are considered promising vehicles for drug delivery due to their biocompatibility and exceptional stability in biological fluids (Akuma et al., 2019). In this aspect, however, it should be stressed that EVs themselves are very complex and thus their components should be taken into consideration as additional active drug constituents (Lener et al., 2015). Furthermore, EVs could be used by pathogens for infection spreading, and thus one can speculate that they may transfer virulence factors, which has to be taken into consideration while manipulating EVs for therapeutic applications (Pironti et al., 2021). On the other hand, 


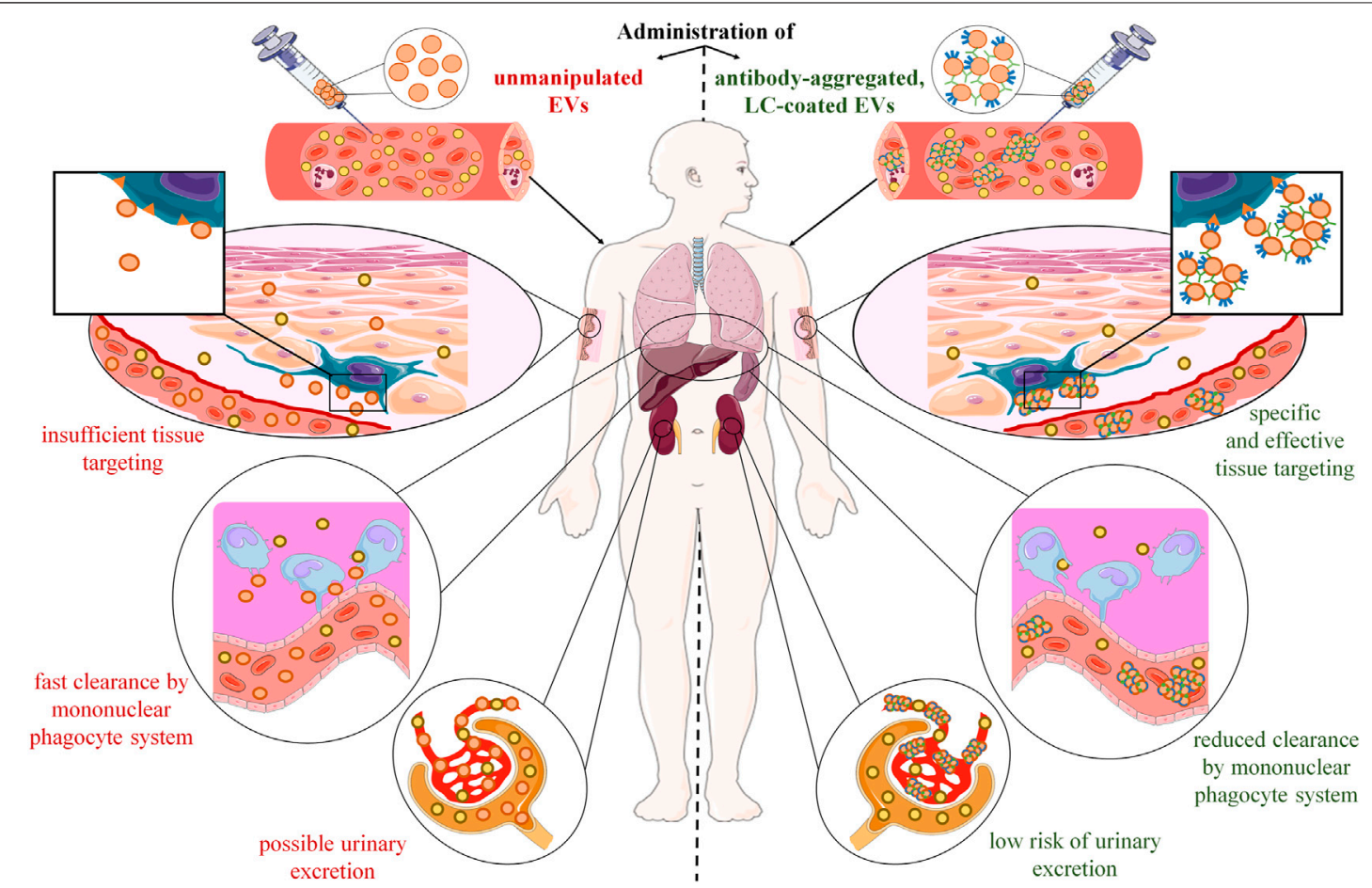

FIGURE 1 | Postulated advantages of extracellular vesicle (EV) aggregation with antigen-specific antibodies and coating with antigen-specific light chains (LC). After systemic administration, therapeutic EVs disperse and mix with their counterparts in the circulation, from which they pass into the tissues. However, their tissue distribution is at least partly random with the preferential accumulation in mononuclear phagocyte-enriched organs, such as liver, spleen, and lungs, where they are rapidly cleared by macrophages. In addition, injected EVs could likely be excreted in urine due to their putative ability to penetrate kidney's glomerular filtration barrier. Altogether, these hurdles make desired tissue penetration and cell targeting insufficient to induce the expected therapeutic effect. However, recent research findings showed that these limitations could be overcome with the use of antigen-specific antibodies and light chains. Incubating EVs with antigen-specific lgG antibodies leads to their aggregation, which enhances their biological efficacy by increasing the amount of EVs that target desired cell population, and by lowering the risk of urinary excretion. In addition, coating EVs with antigen-specific LCs directs them towards target cells, which augments the selectivity of tissue targeting, and limits the unwanted clearance by phagocytes.

attempts to solve the challenges of stem cell therapy allowed to discover that EVs are the main paracrine factors that actually mediate the effects induced by administration of their parental cells (Johnson et al., 2021; Wang et al., 2021). However, despite having many advantages over such approaches, EV-based therapeutics usually achieve lower or almost the same therapeutic efficacy than releasing cells (Kalluri and LeBleu, 2020). Our current research findings suggest that some of these limitations could be overcome by aggregating EVs with antigen-specific antibodies and by increasing the specificity of cell targeting with antibody light chains (LCs). As discussed below, such approaches offer a promising perspective in enhancing EV's therapeutic activity (Figure 1).

\section{"POOR IS THE PUPIL WHO DOES NOT SURPASS HIS MASTER"1}

Recent studies and clinical trials uncovered a number of hurdles that are faced by the cell-based therapies. The

${ }^{1}$ Leonardo Da Vinci (quote translated by Jean Paul Richter) widely described obstacles affecting the efficacy of stem cell therapies result mainly from a lack of standardized treatment procedures, low percentage of cells that reach the desired tissue/organ, the poor survival of engrafted cells that often rapidly undergo apoptosis in targeted tissues, and finally from the diversity of yielded cell populations between individual donors (Hassanzadeh et al., 2021; Johnson et al., 2021; Wang et al., 2021). In addition, stem cell therapies raise concerns about possible adverse events of the treatment that are associated with the risk of tumorigenesis, the possibility of disrupted or abnormal maturation of infused cells as well as with their eventual differentiation in undesirable tissue (Lukomska et al., 2019). On the other hand, the main obstacle related to immunotherapy with dendritic cells results from the risk of their phenotype switching under the influence of the tissue microenvironment, especially at the site of tumorigenesis (Gardner et al., 2020). The risk of losing the desired phenotype may also apply to bone marrow-derived macrophages (Cao et al., 2014), and likely other types of therapeutically administered living cells. Finally, cytokine release syndrome is a major severe complication of the very promising anti-tumor therapy with chimeric antigen receptor (CAR)-T cells that results from the uncontrolled activation of 
recipient cells by transferred CAR-T lymphocytes (Cosenza et al., 2021).

Abovementioned obstacles and concerns prompted researchers to search for safer alternatives that could efficiently replace the living cells for therapeutic purposes. Consequently, EVs released by these cells become considered main promising candidates for cell-free therapies (Tang et al., 2015; Johnson et al., 2021; Wang et al., 2021; Yao et al., 2021).

Along these lines, unlike releasing cell, EVs do not contain all components of cellular machinery required for proper functioning of the living cell, but still they can substitute for its activity. The latter is likely possible due to the contained cargo, which enables EVs to perform a specific function. This assumption is supported by the fact that the cargo is sorted into EVs, exosomes especially, in a very sophisticated manner, and represents a unique content of proteins, RNAs, and lipids, usually differing from the parental cell (Wei et al., 2021). Thus, stem cell-derived EVs cannot differentiate but can promote immune tolerance (Wang et al., 2021), and tissue regeneration (Johnson et al., 2021) instead of tumorigenesis (Lukomska et al., 2019), since they do not carry full genomic DNA material. Moreover, EVs can carry and present antigens as do dendritic cells, but they are unable to switch their phenotype (Yao et al., 2021). Finally, EVs can induce antigen-targeted cytotoxicity against cancer cells without the risk of cytokine release syndrome development (Tang et al., 2015).

It is worth noting that, although EV-based strategies also do not have standardized protocols yet, other concerns and hurdles are either not relevant or could be easily avoided, since EVs are much more manipulable than their parental cells. The latter EV feature together with still expanding knowledge about their biology and functions opened up a new research area attempting both to improve the properties of cell-derived EVs and to design and manufacture their artificial counterparts (García-Manrique et al., 2018) in order to design the most appropriate therapeutic modality, for example in personalized medicine. Such possibilities support the consideration of either manipulated or engineered EVs as next generation biotherapeutics with almost unlimited therapeutic activities and indications that would be easy to produce, handle, and distribute (Johnson et al., 2021). Obviously, as discussed below, selecting the best cellular source of EVs for each considered application is the first crucial step on the way to personalize the EV-based therapeutics (Campanella et al., 2019; Logozzi et al., 2021a). However, before it will happen, researchers have to learn how to improve EVs to surpass both their current therapeutic efficacy and parental cell activities.

\section{CHALLENGES IN THERAPEUTIC APPLICATION OF EXTRACELLULAR VESICLES}

Among other concerns, EV-based approaches avoid the risk of gene mutation, uncontrolled cell division and differentiation as well as immune rejection (Hassanzadeh et al., 2021). Therefore, EVs are considered safer, more controllable as well as much less immunoreactive and immunogenic than the therapeutically administered cells (Buschmann et al., 2021). Other undoubted advantages over the cell-based therapies result from EV's high stability and resistance to harsh conditions in vivo, biocompatibility, ability to cross physiological barriers as well as from their rapid uptake into tissues (Johnson et al., 2021). These features entail a significant increase in interest in the possibility of their therapeutic application, which is still fraught with many challenges (Claridge et al., 2021).

At present, the challenges of standardizing the protocols of EV's generation, characterization, loading with a cargo, dosing and administration are brought to the fore to meet good manufacturing practice requirements (Lener et al., 2015; Buschmann et al., 2021; Thakur et al., 2021). Obviously, choosing the right EV source (i.e., either proper releasing cell subtype or biological fluid), the best loading strategy and the optimal dose administered via the most efficient route would produce the highest efficacy of treatment. But very likely these choices would vary across the specific clinical indications or even patients. The current state-of-the-art technologies and methodologies have been widely summarized elsewhere (Akuma et al., 2019; Doyle and Wang, 2019; Li et al., 2019; Gowen et al., 2020; Klyachko et al., 2020; Gupta et al., 2021; Johnson et al., 2021). However, the use of EV-based therapeutics requires still a very flexible approach for optimization, as it is built on the basis of constantly growing knowledge and clinical evidences.

Although the stem cell therapy achieved promising efficacy with minimal number of reported adverse events in patients with immune-related and inflammatory diseases, stem cell-derived EVs have recently emerged as the easier-to-use treatment modality (Wang et al., 2021) with no risk of stem cell-related coagulopathy (Askenase, 2020) and occlusions (Zhang et al., 2014).

On the other hand, despite promising results in basic and preclinical studies, EVs showed lower than expected therapeutic activity in some cancer clinical trials. Along these lines, dendritic cell-derived EVs demonstrated low clinical efficacy as maintenance immunotherapy in patients bearing inoperable but not progressive non-small cell lung cancer (Besse et al., 2016). This could be due to the fact that EVs were collected from immature dendritic cells and then administered to patients with advanced cancer that likely had impaired immunoreactivity (Yao et al., 2021). This clearly shows that the immune status of patients in target group has to be taken into account while designing appropriate EV-based drug or vaccine.

Another problem is related to pharmacodynamics and pharmacokinetics of therapeutically administered EVs, insufficient tissue penetration and cell targeting especially. Among other possible causes, these limitations could be due to both the dispersion of EVs in biological fluids, and their rapid clearance from circulation by the mononuclear phagocyte system (Figure 1).

Accordingly, after systemic administration, EVs can disperse fast in lymph and blood plasma, where they mix with their counterparts from other cellular sources (Nazimek et al., 2016) and form colloid suspension. Interestingly, EV's colloidal stability in biological fluids seems to be affected by their natural tendency 
to aggregate (Hood et al., 2014). It is worth noting that EV's aggregation could be enhanced by electroporation (Hood et al., 2014), which may impede their further processing for therapeutic application. However, one can speculate that formation of such "colloid aggregates" may increase EV's half-life in circulation by dampening their random passage to tissues. In addition, aggregated EVs would be less likely to overcome the glomerular filtration barrier and become excreted in urine (Erdbrügger et al., 2021). Moreover, it can be hypothesized that EV's aggregation together with directing them towards desired target tissue or cell population will greatly enhance their therapeutic efficacy. As discussed below, our results showed that this could be achieved by incubating EVs with antigen-specific antibodies (Nazimek et al., 2021) (Figure 1).

On the other hand, injected EVs were found to be rapidly cleared by macrophages of the mononuclear phagocyte system. Therefore, they preferentially accumulate in mononuclear phagocyte-enriched organs, such as liver, spleen, and lungs (Chen et al., 2021). Furthermore, resident macrophages are considered to clear EVs also in other tissues. For instance, microglia can phagocytose and thus remove EVs from the brain parenchyma (Schnatz et al., 2021). Thus, fast clearance by macrophages not only hampers EV's delivery to desired tissue and target cell population, but also may either affect the expected therapeutic action or cause some side effects. The latter could be observed if EV's are not cleared in an immunologically silent process but instead when it leads to macrophage activation. Such activation most likely depends on the EV-contained cargo and the state of the donor cell, as discussed recently in the case of microglia (Schnatz et al., 2021). Additionally, one can speculate that macrophages in inflamed tissue are possibly more prone to undergo unexpected activation after EV's engulfment. This seems to particularly apply to the EV-based therapeutics targeting the cells at the site of tissue injury or ongoing inflammation. However, the unwanted clearance could likely be limited by decorating therapeutic EVs with molecules providing "don't eat me" signals (Belhadj et al., 2020), especially in a combination with strategy increasing the specificity and selectivity of cell targeting. And, following the commonly known slogan "simplicity is the ultimate sophistication," antigen-specific antibodies and their derived LCs appear most promising candidates for the latter strategy (Figure 1).

\section{ANTIGEN-SPECIFIC ANTIBODIES AND LIGHT CHAINS IN THE FIGHT TO ENHANCE EXTRACELLULAR VESICLES THERAPEUTIC EFFICACY}

In in vivo conditions, EVs gain most of their attributes at the time of the release by parental cell. However, some of them can be acquired later in the extracellular space. Accordingly, recent study demonstrated the spontaneous formation of a protein corona around EVs in blood plasma (Tóth et al., 2021). Very likely the acquired proteins impact EV's properties and activity, which requires further investigation (Tóth et al., 2021). Similarly, our observations suggested the ability of EVs to bind freely circulating miRNAs (Bryniarski et al., 2015). This could likely be applied in small interfering RNA (siRNA) delivery, as can recently developed EV-liposome hybrid nanoparticles (Evers et al., 2021). These findings also imply that EVs may constitute physiological nanocarriers for removing other molecules from the circulation. Conversely, proteins adhered to EV membrane may redirect the vesicles towards other target.

Along these lines, we found that mouse suppressor T (Ts) cellderived EVs are coated with B1a cell-secreted antibody LCs (Bryniarski et al., 2013; Wąsik et al., 2019; Nazimek et al., 2020). Detailed investigation of this surprising observation allowed us to discover that LC coating ensures the antigenspecificity of Ts cell-released EV's action (Nazimek et al., 2018). Furthermore, coating EVs in vitro with LCs of chosen specificity enables the antigen-specific suppression of immune response (Bryniarski et al., 2013; Nazimek et al., 2018, 2020) (Figure 2). Finally, self-tolerant EVs become capable of suppressing hapten-induced contact hypersensitivity after coincubation with hapten-specific LCs (Nazimek et al., 2019).

We proposed that LCs stick to EV membrane lipids and form multiple adjacent arrangements of high avidity (Nazimek et al., 2016), sufficient for binding to antigenic peptides presented by antigen-presenting cells (APCs) (Nazimek et al., 2020). These findings imply the important role of LCs in specific cell targeting by EVs (Nazimek and Bryniarski, 2020a), thereby increasing their therapeutic potential (Figure 2). Especially that LCs could be coated onto EVs by simple co-incubation, which is a technically easy approach. As mentioned above, protein corona can be acquired by EVs during similar co-incubation with plasma samples (Tóth et al., 2021). Thus, one can assume that the ability to become coated with proteins, including antigen-specific antibodies and LCs, is a general property of EVs that could be widely used for manipulating them for therapeutic purposes. It is also worth noting that the delivery of therapeutic antibodies by EVs can increase their stability and bioactivity, as recently shown in the case of immunoglobulinbearing EVs produced by OKT3 hybridoma (Logozzi et al., 2021b).

Accordingly, considering the immune checkpoint therapy, one can assume that it could be reinforced by coadministration of EV-based therapeutics. In this case, decorating therapeutic EVs with antibodies specific for programmed death receptor-1 ligand (PD-L1) would direct them towards PD-L1-expressing tumor cells, while anticytotoxic T-lymphocyte-associated protein 4 (CTLA-4) antibodies would support regulatory $\mathrm{T}$ cell targeting (Nazimek and Bryniarski, 2020b). Hence, such combined strategy would allow to block immune checkpoints and induce EV-mediated therapeutic effect at the same time, as recently reported (Fan et al., 2021). However, the possibility that therapeutic EVs decorated with anti-PD-L1 antibodies could be bound by PD-L1-expressing tumor EVs to hamper tumor cell targeting cannot be excluded as well, by analogy to the therapies with immune checkpoint inhibitors (Nazimek and Bryniarski, 2020b). Especially that tumor cells are known to potently release EVs into circulation (Logozzi et al., 2021c).

On the other hand, almost 20 years ago it was observed that cross-linking of major histocompatibility complex (MHC) class 


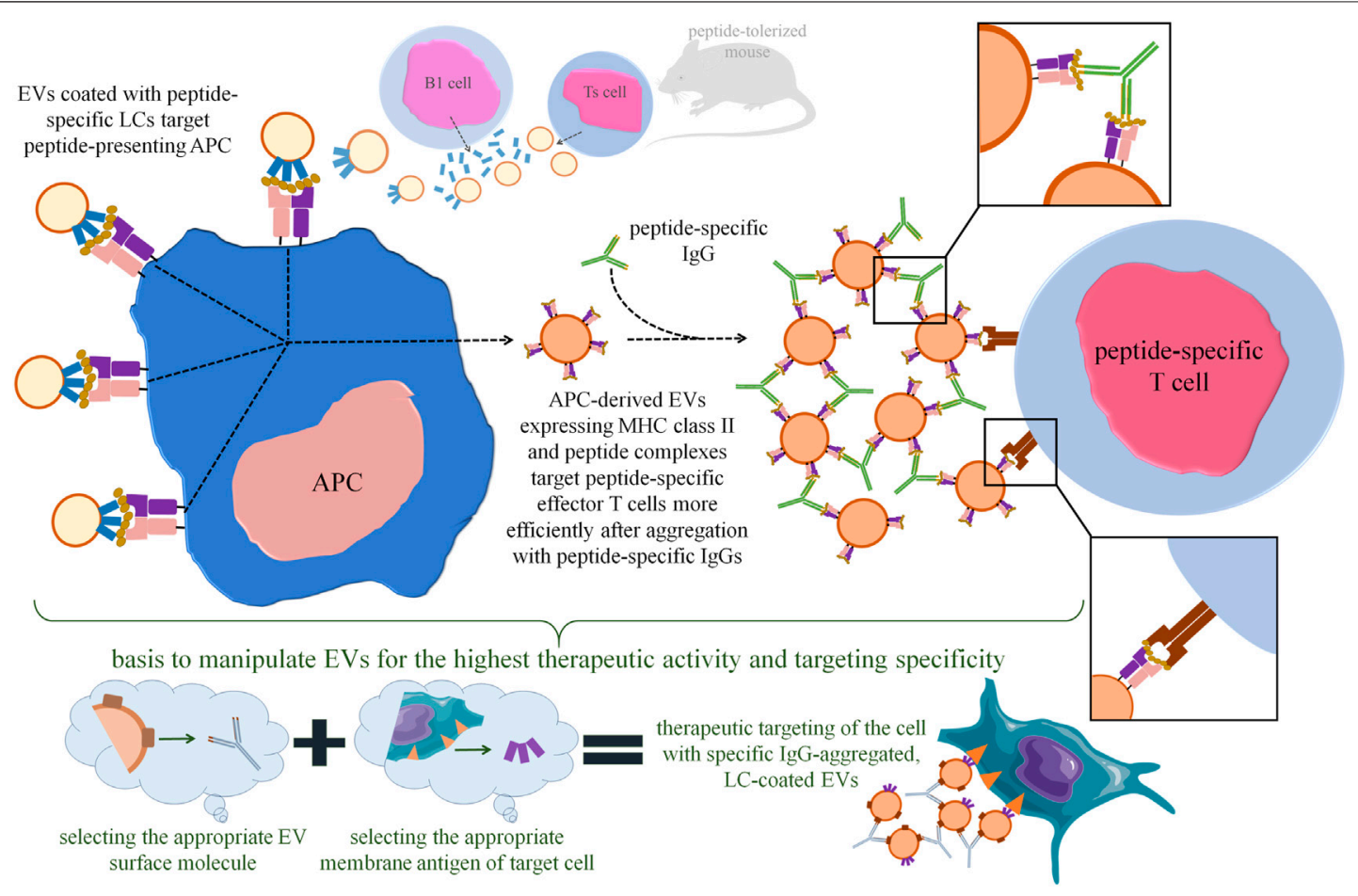

FIGURE 2|Perspectives in increasing the therapeutic efficacy of extracellular vesicles (EVs) with the use of antigen-specific antibodies and light chains (LCs). Our research findings demonstrated that tolerizing mice with antigen-coupled syngeneic erythrocytes activates suppressor $T$ (Ts) cells to release EVs containing immune regulatory miRNA-150. These EVs are then coated with B1 lymphocyte-secreted, antigen-specific LCs, which allows EVs to specifically bind the antigenic peptide presented by antigen-presenting cell (APC). Importantly, Ts cell-derived EVs could also be in vitro coated with LCs of chosen specificity by simple co-incubation. The Ts-cell-EV-targeted APC releases its own EVs expressing major histocompatibility complex (MHC) class II and antigenic peptides that target peptide-specific effector T cells by interacting with T-cell receptor (TCR). Incubation of APC-derived, MHC-expressing EVs with peptide-specific IgG antibodies leads to vesicle aggregation, which greatly enhances their immune suppressive activity by increasing the amount of EVs targeting each effector $T$ cell. Based on these observations, one can assume that therapeutic EVs in general could be manipulated to achieve the highest activity by aggregating them with IgG specific for the selected EV surface molecule, and then by selectively directing them towards target cell with LCs specific for the selected cell surface antigen.

II-expressing EVs with latex beads greatly augments their biological effectiveness (Vincent-Schneider et al., 2002). Similar enhancement was recently demonstrated by us after aggregating MHC class II-expressing EVs with antigen-specific antibodies (Nazimek et al., 2021). These findings imply that EV's aggregation may increase not only their half-life in circulation, as mentioned above, but also the amount of vesicles targeting desired cell population (Figures 1, 2). The latter fits in with the consideration of the optimal EV's dosing at a single cell scale and at the body scale (Gupta et al., 2021). Proposed by us EV's aggregation with antigen-specific antibodies takes an advantage over the latex beads, since antibodies could be safely degraded intracellularly, could limit the unwanted clearance of EVs by phagocytes, and may increase the specificity of cell targeting (Nazimek et al., 2021). Besides, one can assume that EV's could be similarly aggregated with antibodies directed against their surface proteins, which greatly extends the application spectrum of this phenomenon (Figure 2). However, chosen surface molecules bound by aggregating antibodies cannot be involved in EV's cellular uptake to not disturb this process. The latter is implied by the observation that the attempts to use anti-CD9 antibodies abolished the immune activity of EVs (Nazimek et al., 2021).
Additionally, some other aspects of this phenomenon require further investigation. Technically, EV's aggregation could be controlled in vitro with transmission electron microscopy and nanoparticle tracking analysis after standardizing and optimizing the protocols of obtaining the aggregates. Whereas the methods to track the stability and bioactivity of EV's aggregates in in vivo conditions have yet to be developed. Moreover, since aggregation was firstly proved for MHC class II-expressing EVs, the possible MHC-restriction of their activity has to be elucidated with regard to the possibilities to use EVs from allogeneic donors. On the other hand, one can speculate that autologous dendritic cellderived EVs loaded in vitro with dedicated peptides in a direct or indirect manner (Morse et al., 2005) could be considered optimal for aggregation to greatly enhance their vaccine-like activity for instance in cancer immunotherapy (Yao et al., 2021).

\section{CONCLUSION}

Last 2 decades have resulted in tremendous advances in the knowledge of the exceptional functions of EVs, which made them promising candidates for various therapeutic applications. Numerous current research findings have 
provided the basis for attempting to address the challenges faced by such therapies. Herein discussed possibilities to enhance EV's biological activity and specifically direct them towards desired cells with the use of antibodies and LCs constitute crucial steps forward to achieve the highest efficacy of EV-based therapeutics.

\section{DATA AVAILABILITY STATEMENT}

The original contributions presented in the study are included in the article/supplementary material, further inquiries can be directed to the corresponding author.

\section{REFERENCES}

Akuma, P., Okagu, O. D., and Udenigwe, C. C. (2019). Naturally Occurring Exosome Vesicles as Potential Delivery Vehicle for Bioactive Compounds. Front. Sustain. Food Syst. 3, 23. doi:10.3389/fsufs.2019.00023

Askenase, P. W. (2020). COVID-19 Therapy with Mesenchymal Stromal Cells (MSC) and Convalescent Plasma Must Consider Exosome Involvement: Do the Exosomes in Convalescent Plasma Antagonize the Weak Immune Antibodies? J. Extracell. Vesicles 10, e12004. doi:10.1002/jev2.12004

Belhadj, Z., He, B., Deng, H., Song, S., Zhang, H., Wang, X., et al. (2020). A Combined "eat Me/don't Eat Me" Strategy Based on Extracellular Vesicles for Anticancer Nanomedicine. J. Extracell. Vesicles 9, 1806444. doi:10.1080/ 20013078.2020.1806444

Besse, B., Charrier, M., Lapierre, V., Dansin, E., Lantz, O., Planchard, D., et al. (2016). Dendritic Cell-Derived Exosomes as Maintenance Immunotherapy after First Line Chemotherapy in NSCLC. Oncoimmunology 5, e1071008. doi:10.1080/2162402X.2015.1071008

Bryniarski, K., Ptak, W., Jayakumar, A., Püllmann, K., Caplan, M. J., Chairoungdua, A., et al. (2013). Antigen-specific, Antibody-Coated, Exosome-like Nanovesicles Deliver Suppressor T-Cell microRNA-150 to Effector T Cells to Inhibit Contact Sensitivity. J. Allergy Clin. Immunol. 132, 170-181. doi:10.1016/j.jaci.2013.04.048

Bryniarski, K., Ptak, W., Martin, E., Nazimek, K., Szczepanik, M., Sanak, M., et al. (2015). Free Extracellular miRNA Functionally Targets Cells by Transfecting Exosomes from Their Companion Cells. PLoS One 10, e0122991. doi:10.1371/ journal.pone.0122991

Buschmann, D., Mussack, V., and Byrd, J. B. (2021). Separation, Characterization, and Standardization of Extracellular Vesicles for Drug Delivery Applications. Adv. Drug Deliv. Rev. 174, 348-368. doi:10.1016/j.addr.2021.04.027

Campanella, C., Caruso Bavisotto, C., Logozzi, M., Marino Gammazza, A., Mizzoni, D., Cappello, F., et al. (2019). On the Choice of the Extracellular Vesicles for Therapeutic Purposes. Int. J. Mol. Sci. 20, 236. doi:10.3390/ ijms20020236

Cao, Q., Wang, Y., Zheng, D., Sun, Y., Wang, C., Wang, X. M., et al. (2014). Failed Renoprotection by Alternatively Activated Bone Marrow Macrophages Is Due to a Proliferation-dependent Phenotype Switch In Vivo. Kidney Int. 85, 794-806. doi:10.1038/ki.2013.341

Chen, P., Wang, L., Fan, X., Ning, X., Yu, B., Ou, C., et al. (2021). Targeted Delivery of Extracellular Vesicles in Heart Injury. Theranostics 11, 2263-2277. doi: $10.7150 /$ thno. 51571

Claridge, B., Lozano, J., Poh, Q. H., and Greening, D. W. (2021). Development of Extracellular Vesicle Therapeutics: Challenges, Considerations, and Opportunities. Front. Cel Dev. Biol. 9, 734720. doi:10.3389/fcell.2021.734720

Cosenza, M., Sacchi, S., and Pozzi, S. (2021). Cytokine Release Syndrome Associated with T-Cell-Based Therapies for Hematological Malignancies: Pathophysiology, Clinical Presentation, and Treatment. Int. J. Mol. Sci. 22, 7652. doi:10.3390/ijms22147652

Doyle, L., and Wang, M. (2019). Overview of Extracellular Vesicles, Their Origin, Composition, Purpose, and Methods for Exosome Isolation and Analysis. Cells 8, 727. doi:10.3390/cells 8070727

\section{AUTHOR CONTRIBUTIONS}

All authors listed have made a substantial, direct, and intellectual contribution to the work and approved it for publication.

\section{FUNDING}

The article processing charge was covered by the grants number N41/DBS/000419 and N41/DBS/000073 from Polish Ministry of Education and Science. The funder has no role in manuscript preparation and submission.

Erdbrügger, U., Blijdorp, C. J., Bijnsdorp, I. V., Borràs, F. E., Burger, D., Bussolati, B., et al. (2021). Urinary Extracellular Vesicles: A Position Paper by the Urine Task Force of the International Society for Extracellular Vesicles. J. Extracell. Vesicles 10, e12093. doi:10.1002/jev2.12093

Evers, M. J. W., Wakker, S. I., Groot, E. M., Jong, O. G., Gitz-François, J. J. J., Seinen, C. S., et al. (2021). Functional siRNA Delivery by Extracellular VesicleLiposome Hybrid Nanoparticles. Adv. Healthc. Mater., 2101202. doi:10.1002/ adhm.202101202

Fan, Y., Zhou, Y., Lu, M., Si, H., Li, L., and Tang, B. (2021). Responsive DualTargeting Exosome as a Drug Carrier for Combination Cancer Immunotherapy. Research 2021, 1-12. doi:10.34133/2021/9862876

García-Manrique, P., Matos, M., Gutiérrez, G., Pazos, C., and Blanco-López, M. C. (2018). Therapeutic Biomaterials Based on Extracellular Vesicles: Classification of Bio-Engineering and Mimetic Preparation Routes. J. Extracell. Vesicles 7, 1422676. doi:10.1080/20013078.2017.1422676

Gardner, A., de Mingo Pulido, Á., and Ruffell, B. (2020). Dendritic Cells and Their Role in Immunotherapy. Front. Immunol. 11, 924. doi:10.3389/ fimmu.2020.00924

Gowen, A., Shahjin, F., Chand, S., Odegaard, K. E., and Yelamanchili, S. V. (2020). Mesenchymal Stem Cell-Derived Extracellular Vesicles: Challenges in Clinical Applications. Front. Cel Dev. Biol. 8, 149. doi:10.3389/fcell.2020.00149

Gupta, D., Zickler, A. M., and El Andaloussi, S. (2021). Dosing Extracellular Vesicles. Adv. Drug Deliv. Rev. 178, 113961. doi:10.1016/j.addr.2021.113961

Hassanzadeh, A., Rahman, H. S., Markov, A., Endjun, J. J., Zekiy, A. O., Chartrand, M. S., et al. (2021). Mesenchymal Stem/stromal Cell-Derived Exosomes in Regenerative Medicine and Cancer; Overview of Development, Challenges, and Opportunities. Stem Cel Res. Ther. 12, 297. doi:10.1186/s13287-021-02378-7

Hood, J. L., Scott, M. J., and Wickline, S. A. (2014). Maximizing Exosome Colloidal Stability Following Electroporation. Anal. Biochem. 448, 41-49. doi:10.1016/ j.ab.2013.12.001

Johnson, J., Shojaee, M., Mitchell Crow, J., and Khanabdali, R. (2021). From Mesenchymal Stromal Cells to Engineered Extracellular Vesicles: a New Therapeutic Paradigm. Front. Cel Dev. Biol. 9, 705676. doi:10.3389/ fcell.2021.705676

Kalluri, R., and LeBleu, V. S. (2020). The Biology , Function , and Biomedical Applications of Exosomes. Science 367, eaau6977. doi:10.1126/science.aau6977

Klyachko, N. L., Arzt, C. J., Li, S. M., Gololobova, O. A., and Batrakova, E. V. (2020). Extracellular Vesicle-Based Therapeutics: Preclinical and Clinical Investigations. Pharmaceutics 12, 1171. doi:10.3390/pharmaceutics12121171

Lener, T., Gimona, M., Aigner, L., Börger, V., Buzas, E., Camussi, G., et al. (2015). Applying Extracellular Vesicles Based Therapeutics in Clinical Trials - an ISEV Position Paper. J. Extracell. Vesicles 4, 30087. doi:10.3402/jev.v4.30087

Li, X., Corbett, A. L., Taatizadeh, E., Tasnim, N., Little, J. P., Garnis, C., et al. (2019). Challenges and Opportunities in Exosome Research-Perspectives from Biology, Engineering, and Cancer Therapy. APL Bioeng. 3, 011503. doi:10.1063/ 1.5087122

Logozzi, M., Di Raimo, R., Mizzoni, D., and Fais, S. (2021a). What We Know on the Potential Use of Exosomes for Nanodelivery. Semin. Cancer Biol. S1044-579X (21), 00229. doi:10.1016/j.semcancer.2021.09.005

Logozzi, M., Di Raimo, R., Properzi, F., Barca, S., Angelini, D. F., Mizzoni, D., et al. (2021b). Nanovesicles Released by OKT3 Hybridoma Express Fully Active 
Antibodies. J. Enzyme Inhib. Med. Chem. 36, 175-182. doi:10.1080/ 14756366.2020.1852401

Logozzi, M., Mizzoni, D., Di Raimo, R., Giuliani, A., Maggi, M., Sciarra, A., et al. (2021c). Plasmatic Exosome Number and Size Distinguish Prostate Cancer Patients from Healthy Individuals: a Prospective Clinical Study. Front. Oncol. 11, 4258. doi:10.3389/fonc.2021.727317

Lukomska, B., Stanaszek, L., Zuba-Surma, E., Legosz, P., Sarzynska, S., and Drela, K. (2019). Challenges and Controversies in Human Mesenchymal Stem Cell Therapy. Stem Cell Int. 2019, 1-10. doi:10.1155/2019/9628536

Morse, M. A., Garst, J., Osada, T., Khan, S., Hobeika, A., Clay, T. M., et al. (2005). A Phase I Study of Dexosome Immunotherapy in Patients with Advanced Non-small Cell Lung Cancer. J. Transl. Med. 3, 9. doi:10.1186/ 1479-5876-3-9

Nazimek, K., Askenase, P., and Bryniarski, K. (2018). Antibody Light Chains Dictate the Specificity of Contact Hypersensitivity Effector Cell Suppression Mediated by Exosomes. Int. J. Mol. Sci. 19, 2656. doi:10.3390/ijms19092656

Nazimek, K., and Bryniarski, K. (2020a). Approaches to Inducing Antigen-specific Immune Tolerance in Allergy and Autoimmunity: Focus on Antigenpresenting Cells and Extracellular Vesicles. Scand. J. Immunol. 91, e12881. doi:10.1111/sji.12881

Nazimek, K., Bryniarski, K., and Askenase, P. W. (2016). Functions of Exosomes and Microbial Extracellular Vesicles in Allergy and Contact and Delayed-type Hypersensitivity. Int. Arch. Allergy Immunol. 171, 1-26. doi:10.1159/000449249

Nazimek, K., and Bryniarski, K. (2020b). Perspectives in Manipulating EVs for Therapeutic Applications: Focus on Cancer Treatment. Int. J. Mol. Sci. 21, 4623. doi:10.3390/ijms21134623

Nazimek, K., Bryniarski, K., Ptak, W., Groot Kormelink, T., and Askenase, P. (2020). Orally Administered Exosomes Suppress Mouse Delayed-type Hypersensitivity by Delivering miRNA-150 to Antigen-Primed Macrophage APC Targeted by Exosome-Surface Anti-peptide Antibody Light Chains. Int. J. Mol. Sci. 21, 5540. doi:10.3390/ijms21155540

Nazimek, K., Bustos-Morán, E., Blas-Rus, N., Nowak, B., Ptak, W., Askenase, P. W., et al. (2019). Syngeneic Red Blood Cell-Induced Extracellular Vesicles Suppress Delayed-type Hypersensitivity to Self-antigens in Mice. Clin. Exp. Allergy 49, 1487-1499. doi:10.1111/cea.13475

Nazimek, K., Bustos-Morán, E., Blas-Rus, N., Nowak, B., Totoń-Żurańska, J., Seweryn, M. T., et al. (2021). Antibodies Enhance the Suppressive Activity of Extracellular Vesicles in Mouse Delayed-type Hypersensitivity. Pharmaceuticals 14, 734. doi:10.3390/ph14080734

Pironti, G., Andersson, D. C., and Lund, L. H. (2021). Mechanistic and Therapeutic Implications of Extracellular Vesicles as a Potential Link between Covid-19 and Cardiovascular Disease Manifestations. Front. Cel Dev. Biol. 9, 640723. doi:10.3389/fcell.2021.640723

Schnatz, A., Müller, C., Brahmer, A., and Krämer-Albers, E. M. (2021). Extracellular Vesicles in Neural Cell Interaction and CNS Homeostasis. FASEB BioAdvances 3, 577-592. doi:10.1096/fba.2021-00035

Tang, X.-J., Sun, X.-Y., Huang, K.-M., Zhang, L., Yang, Z.-S., Zou, D.-D., et al. (2015). Therapeutic Potential of CAR-T Cell-Derived Exosomes: a Cell-free Modality for Targeted Cancer Therapy. Oncotarget 6, 44179-44190. doi:10.18632/oncotarget.6175
Thakur, A., Ke, X., Chen, Y.-W., Motallebnejad, P., Zhang, K., Lian, Q., et al (2021). The Mini Player with Diverse Functions: Extracellular Vesicles in Cell Biology, Disease, and Therapeutics. Protein Cell. Online ahead of print. doi:10.1007/s13238-021-00863-6

Tóth, E. Á., Turiák, L., Visnovitz, T., Cserép, C., Mázló, A., Sódar, B. W., et al. (2021). Formation of a Protein corona on the Surface of Extracellular Vesicles in Blood Plasma. J. Extracell. Vesicles 10, e12140. doi:10.1002/jev2.12140

Vader, P., Mol, E. A., Pasterkamp, G., and Schiffelers, R. M. (2016). Extracellular Vesicles for Drug Delivery. Adv. Drug Deliv. Rev. 106, 148-156. doi:10.1016/ j.addr.2016.02.006

Vincent-Schneider, H., Stumptner-Cuvelette, P., Lankar, D., Pain, S., Raposo, G., Benaroch, P., et al. (2002). Exosomes Bearing HLA-DR1 Molecules Need Dendritic Cells to Efficiently Stimulate Specific T Cells. Int. Immunol. 14, 713-722. doi:10.1093/intimm/dxf048

Wang, L. T., Liu, K. J., Sytwu, H. K., Yen, M. L., and Yen, B. L. (2021). Advances in Mesenchymal Stem Cell Therapy for Immune and Inflammatory Diseases: Use of Cell-free Products and Human Pluripotent Stem Cell-derived Mesenchymal Stem Cells. Stem Cell Transl. Med. 10, 1288-1303. doi:10.1002/sctm.21-0021

Wąsik, M., Nazimek, K., Nowak, B., Askenase, P. W., and Bryniarski, K. (2019). Delayed-type Hypersensitivity Underlying Casein Allergy Is Suppressed by Extracellular Vesicles Carrying miRNA-150. Nutrients 11, 907. doi:10.3390/ nu11040907

Wei, H., Chen, Q., Lin, L., Sha, C., Li, T., Liu, Y., et al. (2021). Regulation of Exosome Production and Cargo Sorting. Int. J. Biol. Sci. 17, 163-177. doi:10.7150/ijbs.53671

Yáñez-Mó, M., Siljander, P. R.-M., Andreu, Z., Bedina Zavec, A., Borràs, F. E., Buzas, E. I., et al. (2015). Biological Properties of Extracellular Vesicles and Their Physiological Functions. J. Extracell. Vesicles 4, 27066. doi:10.3402/ jev.v4.27066

Yao, Y., Fu, C., Zhou, L., Mi, Q.-S., and Jiang, A. (2021). DC-derived Exosomes for Cancer Immunotherapy. Cancers 13, 3667. doi:10.3390/cancers 13153667

Zhang, B., Yin, Y., Lai, R. C., and Lim, S. K. (2014). Immunotherapeutic Potential of Extracellular Vesicles. Front. Immunol. 5, 518. doi:10.3389/fimmu.2014.00518

Conflict of Interest: The authors declare that the research was conducted in the absence of any commercial or financial relationships that could be construed as a potential conflict of interest.

Publisher's Note: All claims expressed in this article are solely those of the authors and do not necessarily represent those of their affiliated organizations, or those of the publisher, the editors and the reviewers. Any product that may be evaluated in this article, or claim that may be made by its manufacturer, is not guaranteed or endorsed by the publisher.

Copyright (c) 2021 Nazimek and Bryniarski. This is an open-access article distributed under the terms of the Creative Commons Attribution License (CC BY). The use, distribution or reproduction in other forums is permitted, provided the original author(s) and the copyright owner(s) are credited and that the original publication in this journal is cited, in accordance with accepted academic practice. No use, distribution or reproduction is permitted which does not comply with these terms. 